\title{
Zur Rolle der literarischen Lesestrategien bei Lehramtstudierenden. Eine Fallstudie am Beispiel slowenischer Germanistikstudentinnen und -studenten
}

\author{
SAŠA JAZBEC \\ Philosophische Fakultät, \\ Universität Maribor, Slowenien
}

Received: 10 April 2009 / Accepted: 8 September 2009

ISSN: 1697-7467

\begin{abstract}
ZUSAMMENFASSUNG: Der Beitrag zeigt, dass das Wenig-Lesen und Nicht-Lesen in der heutigen multimedial orientierten Gesellschaft ein schwerwiegendes Problem unter Studierenden, genauer gesagt künftigen Lehrpersonen, darstellt. Auf der Basis von Gruppeninterviews wird der Frage nachgegangen, wie die Germanistikstudierenden in Slowenien deutschsprachige literarische Texte lesen bzw. welche Strategien sie dabei einsetzen. Es wird gezeigt, dass die tatsächlichen individuellen Strategien, sowie die erworbenen und nicht erworbenen Kompetenzen nur begrenzt ausreichen. Problematisch ist dies, weil diese individuellen Dispositionen in der späteren beruflichen Betätigung der Lehrpersonen überindividuelle Dimensionen erhalten. Diese sind zunächst zu ermitteln und folglich bei den didaktischen Konzepten stärker zu berücksichtigen. Auf diese Weise können der Literaturunterricht und die Literaturvermittlung gegebenenfalls günstigere Resultate hervorbringen.
\end{abstract}

Schlüsselwörter: Lesen, Studierende, fremdsprachige literarische Texte, Lesekompetenzen

The Role of Literary Reading Strategies for Teacher Trainees.

A Case-Study of Slovene Students of German Studies

\begin{abstract}
The article shows that students or more precisely pre-service teachers are concerned with the problem of little or no reading at all in today's multimedia-oriented society. The research question on how students of German Studies in Slovenia read German literary texts and what reading strategies they apply was investigated in group discussions. The article suggests that the readers' actual individual strategies and their acquired and not acquired competencies are very limited. This presents a problem because these individual dispositions go beyond one's individual proportions required in teaching profession. The latter first ought to be ascertained and later taken into account with regard to the methodological concepts used. In this way literature instruction and literature exchange could yield more favorable results.
\end{abstract}

Keywords: reading, students, literary texts in a foreign language, reading competence 


\section{Einleitung}

Lesen an sich ist ein komplexes Phänomen, das kognitionspsychologisch gesehen einen großen mentalen Aufwand vom Leser verlangt (vgl. Christmann und Groeben, 1999). Beim literarischen Lesen kommen noch andere, textuell bedingte, affektive und subjektive Dimensionen und ihre Wechselwirkungen hinzu. Auf das fremdsprachliche ${ }^{1}$ Lesen wirken darüber hinaus noch weitere Faktoren, sodass mit erhöhter Komplexität gerechnet werden muss. Es wird von verschiedenen Konstanten und Variablen geprägt: 1. von überindividuellen Faktoren wie etwa der Strukturiertheit des Textes sowie 2. von individuellen Faktoren wie den mutter- und fremdsprachlichen Kenntnissen sowie der subjektiven und gesellschaftlich geprägten, ausdifferenzierten literarischen Karriere eines Lesers in der Muttersprache und Fremdsprache.

\subsection{Rahmenbedingungen und Forschungsfrage}

Im (fremdsprachlichen) Literaturunterricht in Slowenien kann man ein Unbehagen seitens der Schüler, aber auch der Lehrpersonen feststellen. Spezielle Leseförderungsmaßnahmen sollen dieses Unbehagen beseitigen, allerdings orientieren sie sich bisher in den meisten Fällen an Schüler/innen. Das Anliegen dieses Beitrags ist es indes, hauptsächlich, auf die Rolle der - in unserem Fall künftigen - Lehrperson hinzuweisen. Es wird davon ausgegangen, dass Lehrpersonen bei der Literaturvermittlung ihre eigenen Lesekompetenzen, ihre Neigung zur und Einstellung gegenüber der Literatur willentlich oder auch unwillentlich, sowohl bewusst als auch unbewusst, weitergeben. Es ist vorauszusetzen, dass das bis dahin Gelesene und frühere Leseerfahrungen der Leser, also sowohl der Lehrpersonen als auch der Schüler, als bewusste oder unbewusste Erinnerung präsent bleiben und den gegenwärtigen und künftigen Umgang mit Literatur beeinflussen und strukturieren (Bonfadelli, 1993; Christmann und Groeben, 1999 u.a.). Versteht man das Lesen als eine kommunikative Handlung, in die der Leser als ein aktives, konstruktives, durch seinen Lebenskontext geprägtes Subjekt eingebunden ist, so lässt sich daraus das Erkenntnisinteresse des vorliegenden Beitrags ableiten: Wie lesen Germanistikstudierende in Slowenien deutschsprachige literarische Texte? Genauer formuliert: Welche Lesestrategien werden beim Lesen fremdsprachiger literarischer Texte tatsächlich eingesetzt? ${ }^{2}$

1. Im Weiteren wird davon ausgegangen, dass fremdsprachige Texte im Prinzip deutschsprachige Texte sind. Wenn von muttersprachigen Texten die Rede ist, dann sind damit slowenische Texte gemeint. In Analogie dazu werden die Syntagmen «fremdsprachliches Lesen» und «muttersprachliches Lesen» verwendet.

2. Angesichts dessen, dass der Kontext dieses Beitrags sehr spezifisch ist (es geht um slowenische Germanistikstudierende und ihre Lesestrategien), kann man die Folgerungen nur begrenzt verallgemeinern. Der Beitrag kann allerdings als eine Basis verstanden werden, die ein erprobtes Untersuchungsinstrumentarium enthält und mit der vergleichbare Untersuchungen in anderen Ländern diskutiert werden könnten. Denn das Schlüsselproblem des (Zu-)Wenig-Lesens ist auch unter Studierenden in anderen Ländern in der heutigen multimedial orientierten Gesellschaft eine aktuelle Herausforderung. 


\section{Erörterung Der LESESTRATEgien}

Strategien, Lesestrategien, literarische Lesestrategien werden hier als ein wichtiger integraler Bestandteil des umfassenderen Konstruktes Lesekompetenz bzw. literarische Lesekompetenz angesehen und verstanden (vgl. Hurrelman und Groeben, 2002). Bei der Reflexion des Begriffes Strategie stößt man sogleich auf einen uneinheitlichen Gebrauch. In der einschlägigen Fachliteratur zum (fremdsprachlichen) Lesen wird und wurde er unterschiedlich verwendet (vgl. Lutjeharms, 1988; Donnerstag, 1989; Ehlers, 1998; Pette, 2001; Denka, 2005). Auf Grund der ausgewählten theoretischen Erörterungen des Strategiebegriffs kann folgendes Fazit gezogen werden: Strategien in der Leseforschung können als die Art und Weise gedeutet werden, in der ein Leser einen Text liest. Sie sind keine Konstrukte, sondern «beschreibbare Lesehandlungen» (Pette, 2001: 296). Dabei kann es sich um bloße technische Handlungen handeln (z.B. den Klappentext lesen, einem Tipp von Freunden folgen, einer reizvollen Titelauffassung nachgehen, Stellen im Text markieren) oder auch um Aktivitäten, die eine Kombination unterschiedlicher, auch nicht spezifisch lesegeprägter Fähigkeiten darstellen, wie etwa das Thematisieren einer Textstruktur, das Suchen von Verknüpfungen zu eigenen Erfahrungen, die Erweiterung von Wissen usw. Ferner können diese Aktivitäten unterschiedlicher Natur sein, d. h. die Bewusstheitskomponente kann verschiedene Stufen aufweisen. Die Bandbreite des Strategierepertoires und die Flexibilität der Strategien, die den Lesern zur Verfügung stehen, können sehr unterschiedlich sein.

Für das Anliegen des vorliegenden Beitrages, der sich auf die literarischen Lesestrategien beim Lesen fremdsprachiger Texte konzentriert, ergibt sich folgende Auffassung:

Lesestrategien sind Handlungen und Aktivitäten, die wir einzeln oder kombiniert beim literarischen Lesen einsetzen. Dazu werden alle affektiv, kognitiv- und motivationsbedingten und praktischen Aktivitäten gezählt. Von Bedeutung ist, dass sie es den Lesern ermöglichen, (fremdsprachige) literarische Texte selbständig zu lesen, ihre sprachlichen, außersprachlichen, expliziten und impliziten, literarischen und fremdsprachlichen Merkmale wahrzunehmen und zu verarbeiten. Außerdem sollen sie es ermöglichen, die Textbedeutung zu rekonstruieren und in ihrem kulturellen Kontext zu verstehen, um diese schließlich in der Diskussion mit anderen Leser/innen reflektieren zu können.

Nach einer Skizzierung des Forschungsansatzes und Untersuchungskontextes der durchgeführten und in diesem Beitrag erläuterten Studie wird den tatsächlich eingesetzten Strategien von Germanistikstudierenden in Slowenien nachgegangen. Da die Fragestellung individuelle, subjektive und detaillierte Begründungen anstrebt, werden die Erkenntnisse mittels qualitativer Forschungsverfahren, im Speziellen in Gruppeninterviews, gewonnen und in der darauf aufbauenden Analyse rekonstruiert und systematisiert. Abschließend werden einige didaktische Folgerungen formuliert.

\section{Feldzugang}

Die literarischen Lesestrategien von Germanistikstudierenden zu untersuchen stellte ein grundlegendes Forschungsinteresse dar, da das individuelle Leseverhalten der Studierenden und künftigen Lehrpersonen bisher nur begrenzt thematisiert wurde (vgl. Schramm, 2001), obwohl es eine maßgebliche Rolle für die spätere Arbeit der Lehrpersonen spielt. Es wird 
davon ausgegangen, dass eine systematische Reflexion der eigenen literarischen Lesesozialisation und der eigenen Lesekarriere, sowie das Bewusstmachen der bereits erworbenen oder potenziellen Lesestrategien, -fertigkeiten und -fähigkeiten das Lesen sowie den Leseprozess der Studierenden in einem neuen Licht erscheinen lässt. Ferner wird vorausgesetzt, dass sich eine reiche Lesesozialisation und stabile Lesegewohnheiten positiv auf die Lektüre (fremdsprachiger) literarischer Texte und somit auch auf den Literaturunterricht auswirken. Des Weiteren kann man den Studierenden im Lehramtsstudium eine gewisse Homogenität unterstellen. Diese ergibt sich aus den vergleichbaren Verpflichtungen während des Studiums im Hinblick auf die spätere pädagogische Tätigkeit in der Schule. Aus diesen Gründen werden den gewonnenen Daten auch allgemeinere Dimensionen unterstellt. Um einerseits der Individualität der Ausführungen Raum zu gewähren und andererseits doch über individuelle Aspekte hinauszugehen, wurden den theoretischen Empfehlungen entsprechend (Lamnek, 1995; Bohnsack, 2003) zwei Gruppen mit acht und sieben Studierenden im letzten Semester ihres Studiums interviewt ${ }^{3}$. Befolgt man das Theoretical sampling nach Glasser/Strauss (1967), dann erfolgt die Auswahl der Probanden nicht nach Kriterien statistischer Repräsentativität, sondern danach, ob sie geeignet sind, das Wissen über den Untersuchungsgegenstand zu erweitern oder nicht. Dazu werden neben den von Morse (1994) vorgeschlagenen Reflexions- und Artikulationsfähigkeiten auch die Faktoren Zeit und Bereitschaft zur Durchführung der Interviews berücksichtigt. Für das Interview lasen alle Beteiligten die zwei Romane «Der Richter und sein Henker» von Friedrich Dürrenmatt und «Demian» von Hermann Hesse. Die Wahl der beiden Romane erwies sich als gelungen, da sie zu unterschiedlichen Reaktionen der Leser und erkenntnisreichen Ergebnissen der Studie führte. Dürrenmatts Roman war für die Studierenden interessant, da sie den Autor nur als Dramenautor kannten und daher mit entsprechenden Erwartungen an die Lektüre herangingen. «Demian» war für die Studierenden dagegen inhaltlich weniger interessant und wurde von ihnen unter Einsatz der Strategien gelesen, die meistens bei Pflichtlektüren ${ }^{4}$ aktiviert werden. Diese stehen zumeist nicht im Einklang mit den Interessen der Studierenden.

Als Erhebungsinstrument wurde das Gruppeninterview ausgewählt, da auf diese Weise Einzelaussagen möglichst effektiv diskutiert werden können. Ferner kann die konkrete Gruppeninterviewsituation, die in dieser Untersuchung thematisiert wird, als eine «natürliche» Situation betrachtet werden, weil die Teilnehmenden - alle sind Germanistikstudierende im achten Semester des Studiums - auch außerhalb der künstlichen Laborsituation eine Gruppe bilden und der Literaturunterricht als eine Variante dieser Forschungssituation begriffen werden kann.

Das Transkribieren der Gruppeninterviews erfolgte nach den Trankriptionsregeln, die von Mayring (1993), Bortz/Döring (1995) und Lamnek (1995) zusammengestellt wurden. Dem

3. Für das Anliegen des folgenden Beitrags wurde nur auf die durchgeführten Gruppeninterviews eingegangen. Es handelt sich so um eine kleine Gruppe, deren Analyse nicht zu repräsentativen, sondern exemplarischen Ergebnissen führt, was bei der qualitativen Forschung immer der Fall ist. Um die gewonnenen Daten zu validieren, wurde in der Studie (Jazbec 2009), auf die wegen des begrenzten Rahmens dieses Beitrags immer nur am Rande eingegangen werden kann, das Triangulationsverfahren eingesetzt. So wurden neun Studierende in Leitfadeninterviews befragt. Diese Interviews wurden anschließend analysiert. Mit einem Drittel zufällig ausgewählten Germanistikstudierenden in Slowenien wurde zugleich eine demoskopische Umfrage geführt (Vgl. Jazbec 2008).

4. Im späteren Verlauf des Beitrages wird darauf näher eingegangen. 
Interview lag darüber hinaus ein schematischer Leitfaden mit Gesprächsimpulsen zu Grunde. Diese beziehen sich einerseits auf den eigentlichen Leseprozess und auf die Erfahrungen, die die Leser dabei machen. Andererseits wird auch das Verstehen des Textes thematisiert, um die indirekt eingesetzten Lesestrategien rekonstruieren zu können.

\subsection{Fallübergreifende Analyse von Gruppeninterviews - das Manual}

Die Auswertung und Analyse einiger ausgewählter Textpassagen erfolgt in drei Schritten. In einem ersten Schritt werden die Transkripte gründlich gelesen. Mittels «Cut-and-PasteTechnik» (Lamnek, 2005:183) werden aus den vorhandenen transkribierten Gruppengesprächsprotokollen diejenigen Stellen ausgewählt, die für die Fragestellung inhaltlich von Bedeutung sind.

In einem zweiten Schritt folgt eine reflektierende Interpretation in Bezug auf die Regeln der deskriptiv-reduktiven Methode. Diese werden einem entwickelten Kategoriensystem (vgl. Jazbec, 2008) zugeteilt, wobei im Rahmen dieses Beitrages lediglich die Kategorie Leseerfahrung dargestellt wird. Der mögliche Vorwurf der subjektiven Selektivität des Materials wird durchgehend berücksichtigt, indem versucht wird, möglichst transparent $\mathrm{zu}$ handeln und textnah $\mathrm{zu}$ argumentieren.

Im abschließenden Auswertungsschritt aller hier abgedruckten und besprochenen Textpassagen werden die rekonstruierten Strategien noch einmal isoliert besprochen.

\subsubsection{Interpretation von Textpassagen}

Im Folgenden werden vier ausgewählte Passagen aus den Gesprächen mit den Gruppennamen A und $\mathrm{B}$ angeführt und analysiert.

\section{Kategorie: Leseerfahrung}

Ausgehend von der offenen Einstiegsfrage Wie war die Lektüre?, die hier der Kategorie Leseerfahrung zugeteilt wird, kommen in allen Gesprächsrunden interessante Aussagen zum Vorschein. Hier werden nur einige angeführt und analysiert.

1. Transkript: Gruppengespräch (Gruppe A) zum Text Der Richter und sein Henker

$1{ }^{5} \mathrm{Bw}$ : ähm, ich weiß nicht, es war, ehrlich gesagt, wir schauen zu viele Filme

2 und zu viel fern und äh als er, in den Wagen, in den Wagen stieg und den Toten

3 ab äh abgefahren hat, dachte ich mir so vom CIA, das kannst du nicht machen [lachen]

4 I: ja, mhm

5 alle: ja, genau [lachen]

6 Hw: ja, ich dachte auch, warum fährt jetzt er fort, ich meine, es ist ein Tatort

7 I: ja und er hat ihn sogar angeschnallt, ist schön beschrieben diese Szene

5. Erläuterung zur Kennzeichnung der Gesprächsteilnehmer: Der Großbuchstabe ist der Anfangsbuchstabe des Namens des Gesprächsteilnehmers oder der -teilnehmerin, w steht für weiblich, m für männlich, I für die Interviewleiterin. 
8 Hw: $\mathrm{mhm}$

9 Bw: es gibt dann keinen Tatort oder der Tatort ist nicht rein, so dass man sehen

10 kann, was dort passiert ist oder ich weiß nicht

$11 \mathrm{Hw}$ : aber man muss auch wissen, das war nicht in der heutigen Zeit, das

12 passierte nicht in der heutigen Zeit, also und äh, und vor ein paar Jahren haben

13 sie die Ermittlungen anders durchgeführt als heutzutage

$14 \mathrm{I}: \mathrm{mhm}$, das ist interessant

$15 \mathrm{Hw}$ : und wahrscheinlich nicht sofort den Tatort gesichert und alles so, wie

16 I: aha, wie wir das aus Filmen kennen

$17 \mathrm{Hw}$ : ja, man muss sich schon äh also vorstellen, dass damals die Ermittlungen

18 anders verlaufen sind als heute

19 I: was hat Sie noch gestört?

$20 \mathrm{Bw}$ : ähm es waren so Abschnitte, es war für mich nicht so zusammengebundene Geschichte

21 Hw: ja, war schon

22 Bw: für mich nicht, nein äh, in einem Abschnitt war, der sollte der Täter sein

23 und das und das ist das und äh in anderen Abschnitt gingen wir dann weiter und

24 äh es gibt, es gab andere Indizien, ich weiß nicht, wie das jetzt zu erklären

25 Hw: aber, wenn man einen Film z. B. ansieht, dann ist das auch, sie zeigen

26 mehrere Personen und auch mehrere Beweise und dann ist es auch nicht ganz

27 klar, so, wer sollte jetzt der Schuldige sein

28 I: das ist wahrscheinlich absichtlich so, denn sonst würden wir den Film nicht sehen

29 Bw: vielleicht war es mir zu kurz und ich konnte mir das nicht so, so vorstellen,

30 es war mir zu schnell, äh

31 I: zu schnell entwickelt sich alles?

32 Bw: ja, ja, zu schnell

33 Aw: kennt ihr die Serie Der Bulle von Tölz? also, als ich das Buch gelesen habe, 34 habe ich immer an ihn gedacht [alle lachen]

Diese Gesprächssequenz trägt, wie auch das gesamte Gespräch, Züge eines kongruenten Gesprächs. Eine Ausnahme stellt lediglich die Interviewte Bw dar, die während der gesamten Zeit eine antithetische Position eingenommen hat. Während sich alle anderen Gesprächsteilnehmenden in dem hier nicht abgedruckten Transkript einig waren, dass die Lektüre im Grunde interessant war, weil es sich um eine nicht allzu umfangreiche Kriminalgeschichte handelte, die schnell und unproblematisch gelesen werden konnte und etwas «anders» war, nahm Bw gewissermaßen die Position eines 'advocatus diaboli' ein. Ihre herausragende Einstellung zu dieser Lektüre, wie auch insgesamt zum Lesen, kam während des ganzen Gesprächs deutlich zum Ausdruck, wobei ihre Argumente und Aussagen (Zeile 29, 30) nicht sehr überzeugend klingen und für die These sprechen, dass sie bewusst die Position der Gegenspielerin in diesem Gespräch einnimmt. Auf der thematischen Ebene fällt auf, dass das Gespräch mit dem Verweis auf Filme zunächst zu einer inhaltlichen Auseinandersetzung mit dem Text führte. Bw stellte fest, dass das Geschehen in «Der Richter und sein Henker» relativ sei, weil es zu sehr den TV-Kriminalserien widerspreche. Dies scheinen für die Interviewten die gängigen Rezeptionsmuster zu sein (Zeile 3 oder Zeile 33-34). Es bestätigt auch die 
Aussage von Hw, die die Feststellung von Bw untermauert, indem sie abweichende Textumstände mit dem Verweis auf eine andere Zeitdimension im Text «plausibel» zu erklären versucht (Zeile 11-13 sowie Zeile 17-18). Hier zeigt sich eine deutlich erkennbare Lesestrategie: das Erklären von Unerwartetem durch Historisieren. Auffallend ist, dass in diesen Aussagen, wie auch im ganzen Gespräch, nichts davon zu spüren ist, dass es sich um einen fiktiven Text handelt und dass dieser die verinnerlichten Muster und vielleicht gesellschaftlich determinierten Regeln tatsächlich und absichtlich stört, indem er sie ironisiert oder sogar absurd darstellt. Obige Aussagen weisen darauf hin, dass dieser literarische Text eher als ein pragmatischer Text oder aber als ein Vertrag zwischen pragmatischem Text und dem Leser gelesen wurde (Gross, 1994). Dies wird auch noch durch das Nichtakzeptieren der Inkohärenz des Textes durch die Leserin (Zeile 20) untermauert. Die Studentin Bw unternimmt einen Versuch, ihre Zweifel an der Kohärenz des Textes zu begründen (Zeile 22-24). Eine Analyse der sprachlichen Formulierung dieser Aussagen deutet daraufhin, dass es vielleicht am Niveau der fremdsprachlichen Kompetenz von Bw liegt, Lücken in der Kohärenz des Textes beim Lesen ausfindig zu machen. Bw exemplifiziert ihre These und relativiert sie dann schließlich mit ihrer durch das ganze Interview transparenten, bereits oben thematisierten Haltung einer Gegenspielerin, indem sie auf den zu geringen Textumfang verweist. Dass dies von den anderen Gesprächsteilnehmern kaum in Betracht gezogen wird, beweist der abrupte Gesprächswechsel von Aw, die spontan auf die Fernsehserie «Der Bulle von Tölz» (Zeile 33-34) verweist und somit das Thema wieder auf das Lesen vor dem Hintergrund des bekannten, durch das Fernsehen geprägten Orientierungsrahmens lenkt.

Auf der Ebene der Interpretation kann festgestellt werden, dass im obigen Abschnitt aus dem Gruppeninterview zwei Themen angesprochen werden. Das erste Thema (Zeilen 1-8, 25-29, 33-34), das im Zusammenhang mit der Fragestellung der vorliegenden Untersuchung als eine Strategie beim Lesen betrachtet wird, ist das Ziehen von Parallelen zu bekannten Filmen. Das zweite Thema (Zeilen 19-24), das eigentlich nur angedeutet wird, aber vermutlich auch als eine literarische Lesestrategie eingesetzt wird, ist, dass beim Lesen Kohärenz auf der Ebene der Geschichte erwartet und nicht etwa konstruiert wird, was bereits seit der Rezeptionsästhetik und auch der Kognitionspsychologie präsumiert wird (Vgl. Iser, 1976; Groeben, 1999 u.a.).

2. Transkript: Gruppengespräch (Gruppe A) zum Text Demian

1 I: also, wie war die Lektüre, wie war DIESE Lektüre? [längere Pause]

2 Nw: irgendwie, keine Ahnung ich hatte es ziemlich schwer diese Sache zu lesen,

3 auf einer Seite war es mir total langweilig, ich hatte keinen Bock drauf, aber dann

4 irgendwie hat es mich so gefesselt, dass ich, wenn ich wirklich angefangen habe $\mathrm{zu}$

5 lesen, ich konnte irgendwie nicht loslassen, obwohl ich äh ständig mir selbst gesagt

6 habe, ich meine, ich hatte ständig das Gefühl, ich mag irgendwie es überhaupt nicht

$7 \mathrm{mehr}$, aber ich konnte trotzdem nicht aufhören, ich weiß nicht wieso, es war

8 irgendwas da drin, was mir [Pause] das Gefühl gegeben hat, ich werde wirklich etwas Neues erfahren, dann wiederum kam das Neue nie

9 I: woran lag das oder liegt das, an der Sprache, an dem Stil oder am Inhalt?

$10 \mathrm{Nw}$ : keine Ahnung, an der Sprache nicht, ich meine, Sprache war mir nicht 
11 irgendwie fremd oder schwer zu lesen überhaupt nicht, aber trotzdem, Inhalt [denkt

12 nach], ja vielleicht, vielleicht Inhalt, ja, vielleicht mehr das, ich meine jetzt nicht

13 insbesondere die einzelnen Geschehnisse, die da waren, sondern, ich hatte

14 irgendwie nicht kapiert, was er damit sagen will

15 Hw: ja, er hat sich eigentlich nie geäußert, was er will, also und dann ständig auch,

16 einige Zeit war er gut, dann war er böse, dann war er wieder gut, dann war ein

17 wieder böse und so, es war eigentlich nicht ganz klar, was er will, also ist es auch

18 schwer so irgendwelche Voraussetzungen zu stellen, ich wusste nicht, was, was soll

19 ich erwarten, also, was wird passieren

$20 \mathrm{I}: \mathrm{mhm}$

$21 \mathrm{Mm}$ : ja also wie gesagt, der Inhalt ist komplex, also dieser ständige Wechsel hin

22 und her die Böse, die Sprache an sich war eigentlich nicht so schwer, es war leicht

$23 \mathrm{zu}$ lesen, aber der Inhalt halt

24 I: war der Inhalt für Sie interessant?

$25 \mathrm{Mm}$ : ja, ich mag eigentlich äh mehr andere Sachen als solche, solche, äh, was ist das

$26 \mathrm{Nw}$ : Psychologie

$27 \mathrm{Mm}$ : ja, genau oder Entwicklungsromane oder so etwas, das mag ich irgendwie nicht so gut

$28 \mathrm{Hw}$ : also ich hatte auch zuerst das Gefühl, also er will irgendwie eine neue Religion

29 einführen, er will sogar den Leser zu dieser Religion irgendwie also auch, so als

30 würde er auf dich wirken wollen also, so dass du auch diese Religion annimmst

$31 \mathrm{Nw}$ : interessant, das habe ich nicht gespürt

$32 \mathrm{Hw}$ : ja, ich schon

33 Nw: mir tat der kleine Junge Leid am Anfang so, ich hatt' ich keinen Bock mehr $\mathrm{zu}$

34 lesen, weil er mir so Leid tat [lacht], der Kleine musste so was erleben, ich meine,

35 so jung, und schon solche Qualen, so neee, doch nicht [lacht]

36 Hw: ja, das war mir auch eigentlich merkwürdig, dass, ich glaube kaum, dass sich

37 zehnjährige Jungen damit beschäftigen, also mit der Religion und also schon, dass

38 sie vielleicht irgendwelche böse Taten machen, aber sie machen sich, sie machen

39 kaum sich wohl größere Gedanken darüber, also, ob das auf ihr gesamtes Leben Einwirkungen haben wird

40 I: $\mathrm{mhm}$, und andere?

41 Tm: also, es war, wegen des Inhaltes schwer zu lesen und man konnte sich schnell

42 verlieren, wenn man so jetzt nicht sehr aufmerksam las, die Sprache war nicht so

43 Inhalt schwierig, aber der halt

44 I: der Inhalt war komplex?

45 Tm: ja

46 I: Am? Entschuldigung [alle lachen, weil sich die Interviewleiterin beim Namen geirrt hat]

47 Dw?. 
48 Dw: für mich auch war das Buch nicht so sehr interessant, ziemlich langweilig,

49 aber die Sprache war leicht und äh ich habe nicht viele Probleme mit der Sprache selbst

50 Aw: ich fand die Sprache auch ziemlich leicht also, es war, es war leicht zu lesen,

51 aber, was mich gestört hat, ist, dass es immer um die Gefühle eigentlich geht, nicht

52 mehr um die Handlung

$53 \mathrm{I}: \mathrm{mhm}$

54 Aw: ich mag mehr die Handlung, deshalb war das nicht interessant für mich

$55 \mathrm{Bw}$ : also mir gefiel das Buch [alle lachen]

56 I: acha, super

$57 \mathrm{Bw}$ : ja, mir war es interessant ein bisschen anders zu lesen als nur, ich weiß nicht,

58 was passiert und wer geht wo und was äh mir war es interessant die Gefühle zu

59 lesen und, ich glaub“ wir alle gingen durch das, was er durchmachte, vielleicht

60 mehr oder weniger, aber ich konnte mich identifizieren mit seinen Gedanken

Die Redebeiträge in diesem Gespräch sind von drei unterschiedlichen Meinungen geprägt. Die meisten Interviewten erfüllten zwar ihre Leseaufgabe, mochten die Lektüre jedoch überhaupt nicht (Zeile 25 und Zeile 48).

Eine Gesprächsteilnehmerin sticht hervor (Zeile 2-8), weil sie eine extrem ambivalente Einstellung der Lektüre gegenüber einnimmt. Sie stellt fest, dass sie einerseits keine Lust dazu gehabt habe, andererseits aber habe sie die Lektüre gefesselt. Sie hatte sich erhofft, beim Lesen WIRKLICH etwas Neues zu erfahren. Was dieses so betonte Neue sein sollte, geht aus dem Interview nicht hervor. Der Versuch der Interviewerin, dem aufgeworfenen Thema nachzugehen, schlug fehl. Nw blieb allerdings in ihren Begründungen weiterhin konsequent. Sie lehnte die Erklärung, sie habe möglicherweise mit der Sprache Probleme gehabt, ab und begründete ihr Nichtverstehen auf der inhaltlichen Ebene (Zeile 12-13).

Die dritte Position der Lektüre gegenüber vertritt Bw (Zeile 55-60), die diese Lektüre im Gegensatz zu allen anderen genossen hatte, wobei sie dies sehr vage argumentiert. Es sei ein bisschen anders zu lesen gewesen. Sie lese sehr emotional orientiert und habe darin Identifikationsangebote gefunden (Zeile 59-60).

Die Argumente, auf denen die zweite und dritte Einstellung von Nw und Bw beruhen, sind als unzureichend zu bewerten. Es stellte sich wieder heraus, dass in dieser Diskussionsrunde zwei «Gegenspielerinnen» mit ihren konträren Einstellungen hervortraten.

Das Unbehagen der meisten Studierenden beim Lesen des Textes «Demian» erklärten sie mit dem uninteressanten, für sie unattraktiven (Zeile 25), schwierigen (Zeile 43-44 und 22-23) Inhalt. Strategisch gesehen relativieren sie das Fremde des literarischen Diskurses mit dem wenig interessanten Inhalt. Der Text verlangte von den Lesern ein aufmerksames Lesen (Zeile 44), was die Fremdsprache nach Karcher (1994) und auch der literarische Diskurs eigentlich voraussetzen (Grosman, 2004), wozu sie aber offensichtlich nicht bereit waren. Dass diese Gruppe den Text sehr ungenau, schnell und unmotiviert las und dass ihre drei unterschiedlichen Positionen nicht mit überzeugenden Argumenten untermauert sind, bestätigt u. a. auch Folgendes: Aufgrund technischer Mängel waren beim Kopieren des Textes an drei Stellen Seiten ausgelassen worden, was keinem aus dieser Gruppe beim Lesen auffiel. Darüber hinaus brachten die Redebeiträge aller Gesprächsteilnehmenden ihre selbstbewusste Haltung bezüglich ihrer fremdsprachlichen Kenntnissen zum Ausdruck (Zeile 10-11, 22, 43-44, 48-49). Dass das 
sprachliche Fremde in literarischen Texten präsupponiert wird und dass ein solches Unbehagen bei kompetenten, sprachlich sensibilisierten wie auch bei muttersprachlichen Lesern zum literarischen Lesen gehört, ist diesen Interviewten offenbar nicht bewusst gewesen.

Im Hinblick auf die strategischen Handlungen kann schließlich zusammengefasst werden, dass die Gesprächsteilnehmer/-innen beim Lesen diejenige Strategie einsetzten, die bei der Pflichtlektüre üblich ist: Sie lesen einen inhaltlich unattraktiven Text (Pflichtlektüre) schnell und oberflächlich durch, um die Aufgabe zu erledigen. Zweifellos ist diese Strategie keine literarische Strategie bzw. hat mit dem eigentlichen literarischen Lesen nichts gemein.

\section{FAZIT}

Aus allen hier abgedruckten und besprochenen Textpassagen kann das folgende Strategienspektrum rekonstruiert werden:

- Ziehen von Parallelen mit bekannten Filmen;

- Erwarten von Kohärenz auf der Ebene der Geschichte;

- Erklären von Unerwartetem durch Historisieren;

- Schnelles und oberflächliches Lesen eines inhaltlich unattraktiven Textes (Pflichtlektüre) zur Erledigung der Aufgabe;

- Einebnen der Komplexität des Textes statt konstruktive Verarbeitung;

- Relativieren des Fremden des literarischen Diskurses mit dem wenig interessanten Inhalt;

- Verzögertes, konzentriertes, mehrmaliges Lesen.

Die ermittelten Strategien dokumentieren einen betont individualisierten Lesevorgang. Die Studierenden betrachteten die Leseaufgaben als Pflicht und setzten beim Lesen Strategien ein, die sie als Germanistikstudierende im Laufe ihrer Lesesozialisation entwickelt haben. Man kann sie als ein «sich durch einen (anspruchsvollen) fremdsprachlichen literarischen Text Hindurchkämpfen» zusammenfassen. Sie gehen auf die Pflichtlektüre ein und versuchen zunächst ihren eigenen Erfahrungsschatz zu aktivieren, wobei sie viele «Erfahrungen» offensichtlich mithilfe anderer Medien, hier vor allem durch das Fernsehen sammeln. Werden Anknüpfungspunkte gefunden, wie dies bei «Der Richter und sein Henker» deutlich wurde, dann erwartet man eine kohärente Geschichte und empfindet alle Abweichungen, Leerstellen sowie detaillierten Beschreibungen im Text als störend. Die Orientierung an linear ablaufenden Handlungssträngen beeinflusst entscheidend den Leseakt, indem man ihn nur auf eine Dimension beschränkt. Man liest involviert, schnell und in Extremfällen legt der Leser den Text nicht mehr zur Seite. Für das Lesen im Sinne Eggerts - als einem Bestandteil eines Prozesses, in dem Anregungen von außen angenommen und in eigenständiger Weise verarbeitet, für sich selbst formuliert bzw. mit kommunikativer Absicht nach außen getragen werden (Eggert, 2004:112), bleibt weder Zeit noch Lust oder Energie - geschweige denn Bedarf. Aus den Gesprächen lässt sich ableiten, dass die literarischen Lesestrategien, die beim Lesen eines fremdsprachigen literarischen Textes eingesetzt werden müssten, den Studierenden zumindest in Ansatzpunkten bekannt sind.

Schließlich bleibt in diesem Zusammenhang noch zu erwähnen, dass in den Gesprächen deutlich wurde, dass die Studierenden beim Lesen zwar ihre bereits existierenden Erfahrungen 
aktivieren, offensichtlich aber keine Bereitschaft und keinen Bedarf zeigen, ihre Erfahrungen beim und nach dem Lesen eventuell in Frage zu stellen, zu erweitern u.a.m.

Der Lesevorgang bei einem literarischen Text, der den Studierenden wenig oder sogar völlig uninteressant erscheint, wie dies bei dem Roman «Demian» der Fall ist, verläuft unter der Prämisse der Individualisierung der Lektüre, obwohl es hier im Vergleich zu «Der Richter und sein Henker» offensichtlich weniger Anknüpfungspunkte für die Studierenden gibt. Sie nehmen zwar die Komplexität des wenig handlungsreichen Textes wahr, aber statt zu versuchen, diese zu verarbeiten, gehen sie einen anderen Weg. Sie lockern die Komplexität, indem sie auf den uninteressanten Inhalt verweisen und rechtfertigen damit ein flüchtiges, oberflächliches Lesen. Ferner deutet das Gespräch in einigen Teilen darauf hin, dass bei den Studierenden die Gleichsetzung von Geschichte und Realität sowie von Autor und Erzähler nicht überwunden ist. «Demian» wird beispielsweise für sie hauptsächlich zum Träger von Charakterzügen Hesses.

\subsection{Folgerungen für die Literaturdidaktik}

Aus den hier präsentierten Ergebnissen, sowie aus der umfassenderen Studie lassen sich einige Folgerungen für die (fremdsprachliche) Literaturdidaktik ableiten. Diese sind in Slowenien unmittelbar interessant, anderswo mittelbar, falls dort vergleichbare Umstände in der (fremdspachlichen) Literaturdidaktik festgestellt werden können. Die Ergebnisse können somit als Impulse für ähnliche oder andersartige literaturdidaktische Schlussfolgerungen fungieren.

Die Analyse der Ergebnisse weist darauf hin, dass sowohl die muttersprachliche als auch die fremdsprachliche Literaturdidaktik die existierenden Konzepte überdenken muss. Das bisherige Konzept, das sich auf die unterrichtsbezogene Situation und Literaturvermittlung konzentrierte, reicht nicht aus. Vielmehr hat sich seine Wirkung bei den meisten Interviewten sogar als kontraproduktiv erwiesen. Der Schlüsselkompetenz Lesen und dem Leseprozess sollte man in allen Schulfächern mehr Aufmerksamkeit schenken. Darüber hinaus sollte sie intensiv gefördert werden. Für den Literaturunterricht erfordert das nicht nur die Auseinandersetzung mit dem eigentlichen literarischen Lesen, sondern auch die Thematisierung des literarischen Lesens auf der Metaebene. Ferner impliziert ein solches Vorgehen auch die Ermittlung der Lesebiographien und die Diskussionen der unterschiedlich entwickelten Lesekarrieren einzelner Leser/innen. In der Lehrerausbildung sollten sich die Studierenden daher intensiver mit empirischen, sowohl qualitativen als auch quantitativen, Methoden der Leserforschung im Rahmen des Studiums beschäftigen, um sie später erfolgreich einsetzen zu können.

Die empirisch ermittelten Lesebiographien können u. a. die Kompetenzen und Strategien der Leser, Wenigleser und Vielleser, die sie im Umgang mit anderen Medien entwickeln, zu Tage fördern. Diese Ergebnisse können gegebenenfalls die Literaturdidaktik bereichern. Der Literaturunterricht würde sich somit nicht nur oder vorwiegend auf Printtexte konzentrieren, sondern auch auf andere mediale Formen der Literatur. Die Kompetenzen und Strategien der jungen Leser/-innen sind in diesen Bereichen vielleicht ausgeprägter und es erscheint sinnvoll, von den schon vorhandenen Kompetenzen auszugehen, diese und deren Transfer zu fördern oder neue Kompetenzen zu entwickeln.

Eine weitere Aufgabe des Literaturunterrichts ist der Vergleich von literarischem und pragmatischem Lesen. Ein unabdingbarer Bestandteil des zukünftigen Literaturunterrichts muss sein, den Rahmen, innerhalb dessen man literarische oder pragmatische Texte liest, mit den Lernern zu besprechen und somit auch die problematische Trennung zwischen Literatur und 
Nicht-Literatur, zwischen Fiktivem und Realem, zu thematisieren.

Die Literaturdidaktik in Slowenien darf das Ergebnis der Analyse, dass hauptsächlich emotionale Gratifikationen vom literarischen Lesen erwartet werden, nicht umgehen. Zwar sollte der Literaturunterricht den Lesern diese Gratifikationen gewähren, zugleich sollte er ihnen aber auch die literarischen Diskrepanzerfahrungen ermöglichen, die zu einer Erweiterung und Ausdifferenzierung ihrer bisherigen Strategien beitragen können.

Die Berücksichtigung dieser Implikationen könnte die Literaturdidaktik neu positionieren und folglich auch die Leseförderung in institutionellen Kontexten begünstigen.

\section{LITERATURVERZEICHNIS}

Bohnsack, R. (2003). «Gruppendiskussion», in U. Flick und E. Kardorff, I. Steinke (Hrsg.). Qualitative Forschung. Ein Handbuch. Hamburg: Rowohlt, 369-384.

Bonfadelli, H. u.a. (Hrsg.) (1993). Leseerfahrungen und Lesekarrieren. Gütersloh: Verlag Bertelsmann Stiftung

Bortz, J. und Döring, N. (1995). Forschungsmethoden und Evaluation für Sozialwissenschaftler. Heidelberg: Springer.

Christmann, U. \& Groeben, N. (1999). «Psychologie des Lesens», in B. Franzmann, K. Hasemann, D. Löffler, E. Schön, (Hrsg.), Handbuch Lesen. München: Saur, 145-223.

Denka, A. (2005). Lesestrategien und Lesesteuerungsstrategien beim Einsatz literarischer Texte im Fremdsprachenunterricht. Frankfurt am Main: Peter Lang.

Donnerstag, J. (1989). Rezeptionstheorie und Literaturdidaktik. Literarische Lesestrategien und rock lyrics im Englischunterricht. Essen: Die blaue Eule.

Dürrenmatt, F. (1971). Der Richter und sein Henker. Hamburg: Rororo.

Eggert, H. (2004). Lügen und ihre Widersacher. Würzburg: Königshausen \& Neumann.

Ehlers, S. (1998). Lesetheorie und fremdsprachliche Lespraxis. Tübingen: Gunter Narr Verlag.

Glasser, B., Strauss, A. (1967). The Discovery of Grounded Theory: Strategies for Qualitative Research. Chicago: Aldine.

Groeben, N. (Hrsg.) (1999). Lesesozialisation in der Mediengesellschaft. Ein Schwerpunktprogramm. 10. Sonderheft: IASL. Tübingen: Niemeyer.

Groeben, N., Hurrelmann B. (Hrsg.) (2002). Lesekompetenz. Bedingungen, Dimensionen, Funktionen. Weinheim u. München: Juventa.

Gross, S. (1994). Lese-Zeichen: Kognition, Medium, Materialität im Leseprozess. Darmstadt: Wissenschaftliche Buchgesellschaft.

Grosman. M. (2004). Zagovor branja.[Die Verteidigung des Lesens]. Ljubljana: Sophia.

Hesse, H. (1974). Demian. Frankfurt am Main: Suhrkampf.

Iser, W. (1976). Der Akt des Lesens. München: Wilhelm Fink.

Jazbec, S. (2008). Lesestrategien beim Lesen fremdsprachiger Literatur. Eine Untersuchung am Beispiel slowenischer Germanistikstudentinnen und -studenten. Dissertationsschrift. Universität Ljubljana.

Jazbec, S. (2009). Man taucht in eine andere Welt ein... Lesestrategien beim Lesen fremdsprachiger Literatur. Eine Untersuchung am Beispiel slowenischer Germanistikstudentinnen und studenten [erscheint 2009 im Peter Lang Verlag].

Lamnek, S. (1995). Qualitative Sozialforschung. Bd 1: Methodologie. Weinheim: Beltz.

Lamnek, S. (2005). Gruppendiskussion. Weinheim: Beltz.

Lutjeharms, M. (1988). Lesen in der Fremdsprache. Bochum: AKS-Verlag. 
Mayring, P. (1993). Qualitative Inhaltsanalyse - Grundlagen und Techniken. Weinheim: Deutscher Studien Verlag.

Morse, J. M. (1994). «Designing Funded Qualitative Research», in N. Denzin, Y. Lincoln (Hrsg.), Handbook of Qualitative Research Thousand Oaks: Sage, 220-235.

Pette, C. (2001). Psychologie des Romanlesens. Lesestrategien zur subjektiven Aneignung eines literarischen Textes. Weinheim und München: Juventa Verlag.

Schramm, K.(2001). L2-Leser in Aktion. Der fremdsprachliche Leseprozeß als mentales Handeln. Münster: Waxmann Verlag. 